\title{
Implementation of multitasking on a small microcomputer
}

\author{
LLOYD HASTINGS and N. DEREK ARNOLD \\ University of Cincinnati, Cincinnati, Ohio
}

\begin{abstract}
A kernal program written in 6502 assembly language that allows as many as oix different assembly language programs to run concurrently on a single small microcomputer is described. The basic principle used is that of time division multiplexing to allow multitasking. This is accomplished by assigning each program its own stack area and allowing it to execute for a specified duration, as determined by an interrupt-driven master program. This interrupt program also controls the proper sequencing of the programs and maintains the functional integrity of each individual program.
\end{abstract}

Although microcomputers have continued to increase in speed and power and to decrease in cost, the widespread use of such computers in psychology laboratories has been limited by the devlopment and availability of appropriate software. However, as time progresses, more and more software is being generated. Recent reports (Butler, 1980; Carroll, Santi, \& Rudell, 1981) have described software packages that are both versatile and easy to use. One disadvantage is that they are designed primarily to control only one experimental station. Programs that have been written to handle multiple stations and/or different programs (Ternes, Ehrman, \& O'Brien, 1982) usually require large amounts of memory and expensive peripherals, that is, disk drives.

Numerous methods exist that permit a microcomputer to run a number of programs concurrently. These differ in many ways, including speed of execution, complexity of operation, and related factors. Probably the most sophisticated approach is that of multiprogramming, defined by Kaplan (1980) as "the division of a program into several asynchronous parts, controlled by external interrupts" (p. 137). Usually, the different parts or tasks are assigned a certain priority, with those of a higher priority being run before or instead of the lower priority tasks. Conceivably, then, some programs may never be executed or be executed only rarely if higher priority events occur frequently. Such an arrangement would clearly be unacceptable in a situation in which all tasks were of the same priority: for example, if in a typical psychological experiment a computer may be controlling four separate test stations, each running a different operant conditioning paradigm, and the detecting of responses, timing out of events, etc., would be of equal importance for all four tasks. In this instance, a special case of multiprogramming, called multitasking (or timesharing), would be more appropriate. In multi-

The authors' mailing address is: Department of Environmental Health, College of Medicine, University of Cincinnati, Cincinnati, OH 45267. tasking, each task is guaranteed an equal percentage of central processor unit (CPU) time during each second of real time. Thus, a number of logically independent subprograms can be set up to pass control of the CPU from one to another in a sequential, or "round-robin," fashion. Since these subprograms share the CPU in an equal fashion, Wyss (1983) suggested that they be called coroutines, rather than subroutines. A further distinction is that a coroutine can relinquish control of the CPU at any point in its execution and then resume at that point when it regains control; in contrast, subroutines are initiated at their beginning every time they are called.

The following describes a kernel program written in 6502 assembly language that allows as many as six different assembly language programs to run concurrently on a single small microcomputer. In general, the complexity of the programs is limited mainly by the availability of memory and input/output $(\mathrm{I} / \mathrm{O})$ ports. In addition, certain limitations that prevent some types of programs from being run exist. For example, programs that analyze data in real time or transfer data to disk storage would be difficult to implement due to timing considerations. However, these concerns are not of paramount importance for many studies that involve the collection of behavioral data (e.g., schedules of reinforcement, avoidance paradigms, etc.).

A major feature of this software design is that the individual programs can be written in a straightforward manner and be completely independent of each other, that is, as if each program was the only program being run. This greatly reduces the complexity of the programs and results in a decrease in expenditure of programming effort.

The program was developed to run on the Ohio Scientific C1P microcomputer, but can be implemented on any microcomputer using the $6502 \mathrm{CPU}$. The basic principle used is that of time division multiplexing to allow multitasking. Basically, this is accomplished by giving each program its own stack area and allowing it 
Table 1

Stack Allocation

\begin{tabular}{|c|c|c|c|c|}
\hline Program No. & Program Name & $\begin{array}{c}\text { Stack Location } \\
\text { (Base 16) }\end{array}$ & $\begin{array}{l}\text { Stack Initialization } \\
\text { (Base 16) }\end{array}$ & Start of Program \\
\hline 0 & Cage 1 & $\$ 0100-\$ 0127$ & $\$ 21(\$ 0121)$ & $\$ 0500$ \\
\hline 1 & Cage 2 & $\$ 0128-\$ 014 \mathrm{E}$ & $\$ 48$ & $\$ 0600$ \\
\hline 2 & Cage 3 & $\$ 014 \mathrm{~F}-\$ 0176$ & $\$ 70$ & $\$ 0700$ \\
\hline 3 & Cage 4 & $\$ 0177-\$ 019 E$ & $\$ 98$ & $\$ 0800$ \\
\hline 4 & SUBMON & $\$ 019 F-\$ 01 C 6 *$ & $\$ C 0^{*}$ & $\$ 0900$ \\
\hline 5 & PROMPT & $\$ 01 C 7-\$ 01 E E$ & $\$ E 8$ & $\$ 0 \mathrm{~A} 00$ \\
\hline 6 & FILL & \$01EF-\$01FF & $\$ F 9$ & $\$ 0 \mathrm{~B} 00$ \\
\hline
\end{tabular}

*In OSI microcomputers, the IRQ jump vector is stored at \$01C0-\$0IC2; consequently, this "mini-stack" should stop at \$01BF, with initialization at \$01B9.

to be executed for a specified duration (time division multiplexing), as determined by an interrupt-driven master program. The use of a timed interrupt to pass control from one program to another is called "time slicing" (Holtzer, 1983). The general concept has been used previously to implement multitasking programs (Brown, 1981). It is presented here in a configuration that allows execution of programs in real time, since many applications in psychology have this as a requirement.

The fundamental technique that makes multitasking possible is manipulation of the stack pointer during an IRQ interrupt. When a IRQ interrupt occurs, the CPU forces the current address onto the stack and then jumps to the interrupt program. After the interrupt has been serviced and the CPU has executed a returnfrom-interrupt (RTI) instruction, the opposite occurs. The address is removed from the stack and the program resumes at the address following the last instruction executed prior to when the interrupt took place. However, if the starting address of another program is forced onto the stack before the RTI is executed, then the microcomputer will branch to this second program and begin running it. This procedure, along with provisions for keeping track of which program is to be run next and preserving the operating environment $(\mathrm{X}, \mathrm{Y}$ registers, etc.) of each program, constitutes a key feature of the multitasking program described in this paper.

Five major steps are necessary in order to implement multitasking on a 6502-based microcomputer. These include (1) stack allocation, (2) setting up a stack pointer table, (3) loading the stack with the starting address of each individual program, (4) setting up an interrupt-driven IRQ program to keep track of the sequence of the programs being run, and (5) initialization of a clock to generate the interrupt.

The stack of the 6502 resides at addresses $\$ 0100$ through \$01FF. It operates in a "last in, first out" manner, with the Stack Pointer register initially containing $\$ F F$ ( $\$ 01 F F$ ). Thus, when the 1st byte is pushed onto the stack, the byte is stored at $\$ 01 F F$ and the Stack Pointer is automatically decremented to $\$ F E$. The first step in setting up the stack for multitasking is to divide the stack area into a specified number of "mini- stacks," one for each program to be run. In the example presented here, the stack area has been divided into six "mini-stacks," each 40 bytes in length (with some residual space-see Table 1). As an illustration, the "mini-stack" for Program No. 0 occupies addresses $\$ 0100-\$ 0127$ (see Table 2). During an IRQ, 3 bytes are used automatically, beginning with $\$ 0127$, to store Program Counter High (PCH), Program Counter Low (PCL), and the Processor Status Register (PS). Three more are used by the IRQ program to store the Accumulator (A) and the $X$ and $Y$ registers. At this point, the Stack Pointer is pointing to the 7th byte of the "ministack," or \$0121. Whenever this address is placed into the Stack Pointer and an RTI is executed, the registers would be restored and the program residing at $\$ 0127$ (PCH) and \$0126 (PCL) run. By storing the address of the 7th byte of each "mini-stack" in a table (see Tables 3 and 4) and then loading these addresses into the Stack Pointer during each interrupt in a sequential manner, a different program would be run after each RTI.

After the stack has been allocated, and the Stack Pointer table set up, each location in the "mini-stack" designated PCH, PCL must be loaded with the starting address of each individual program (see Table 5). Starting addresses are completely arbitrary and depend only on where each program is placed in memory (e.g., Program No. 0 begins at $\$ 0500$ ).

It is quite apparent that complete control over the stack is necessary at all times. This is handled by the

Table 2

\begin{tabular}{ccc}
\multicolumn{3}{c}{ Program No. 0: “Mini-Stack” } \\
\hline Location & Stack & Contents \\
\hline$\$ 0127$ & PCH & $\$ 05$ \\
0126 & PCL & $\$ 00$ \\
0125 & PS & \\
0124 & A & \\
0123 & Y & \\
0122 & X & \\
0121 initialized & & \\
& & \\
$-\quad$ & & \\
- & & \\
&
\end{tabular}


Table 3

Stack Pointer Table

\begin{tabular}{cc}
\hline Location & Contents \\
\hline$\$ 000 \mathrm{C}$ & $\$ 21$ \\
$\$ 000 \mathrm{D}$ & $\$ 48$ \\
$\$ 000 \mathrm{E}$ & $\$ 70$ \\
$\$ 000 \mathrm{~F}$ & $\$ 98$ \\
$\$ 0010$ & $\$ C 0^{*}$ \\
$\$ 0011$ & $\$ \mathrm{E} 8$ \\
$\$ 0012$ & $\$ \mathrm{F9}$ \\
\hline
\end{tabular}

*For OSI, this value should be $\$ B 9$.

Table 4

Assembly Language Listing of Program to Create Stack Pointer Table for the Seven "Mini-Stacks"

\begin{tabular}{|c|c|c|c|}
\hline $\begin{array}{l}360 \\
370 \\
380 \\
390 \\
400 \\
410 \\
420 \\
430 \\
440 \\
450 \\
460 \\
470 \\
480 \\
490 \\
500 \\
510 \\
520 \\
530 \\
540 \\
550\end{array}$ & $\begin{array}{l}\text { LDX } \\
\text { TXB } \\
\text { LDA } \\
\text { STA } \\
\text { STA } \\
\text { LDA } \\
\text { STA } \\
\text { LDA } \\
\text { STA } \\
\text { LDA } \\
\text { STA } \\
\text { LDA } \\
\text { STA } \\
\text { LDA } \\
\text { STA } \\
\text { LDA } \\
\text { STA } \\
\text { LDA } \\
\text { GTA }\end{array}$ & 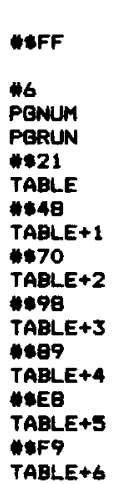 & $\begin{array}{l}\text { INITIALIZE } \\
\text { SET UP TO BTART BYSTEM BY BTART- } \\
\text { INS AT PROB } 6 \\
\text { BET TO RUN PROC } 6 \\
\text { (INTERRUPT PROGRAM () } \\
\text { (LABT PROGRAM RUN IN MUTIPLEX) } \\
\text { BET UP BTACK POINTER TABLE } \\
\text { ( TABLE-\$OOOC ) }\end{array}$ \\
\hline
\end{tabular}

Table 5

Assembly Language Listing of Program to Initialize Starting Addresses in Each "Mini-Stack"

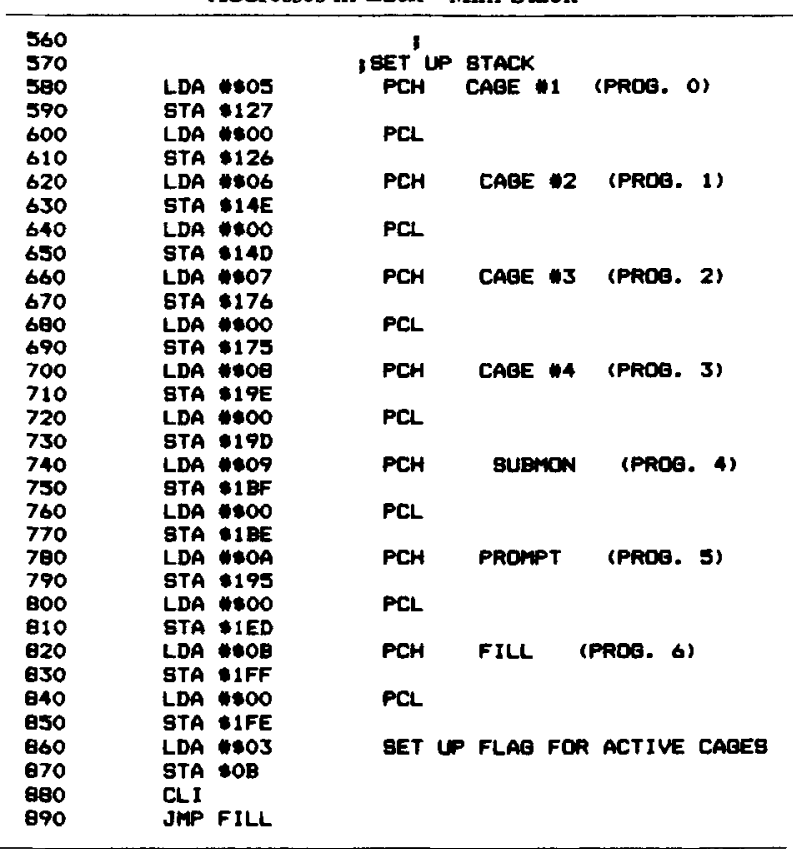

IRQ multiplexing program flowcharted in Figure 1 (also see Table 6). The status of each program at the time it is interrupted is stored until its turn comes up again, at which time its execution is resumed and the updated status stored once more. The programs are run in a sequential manner, as called up from the Stack Pointer table. If there is no program to run, provision has been made to skip to a dummy program (FILL). This program consists simply of a jump instruction to a jump instruction, which causes the program to loop continuously.

The final step is to set up a clock that generates the IRQs. This is easily accomplished by using the T1 timer on a 6522 VIA. The Stack Pointer should also be set at \$FF so that at the first interrupt, Program No. 0 will subsequently be executed.

This completes the steps necessary for setting up the stack in order to implement multitasking on the 6502 . To this point, no application programs have been considered. Although these can be of almost any nature, the labels in this example suggest that the application programs might consist of four programs to control four experimental cages plus two auxiliary programs-one to accept keyboard entry (PROMPT) and one to update the display screen (SUBMON). A seventh, FILL, consists only of a JMP (jump) statement to produce a loop.

As noted earlier, each program resides in its own separate and independent block of memory. Further-

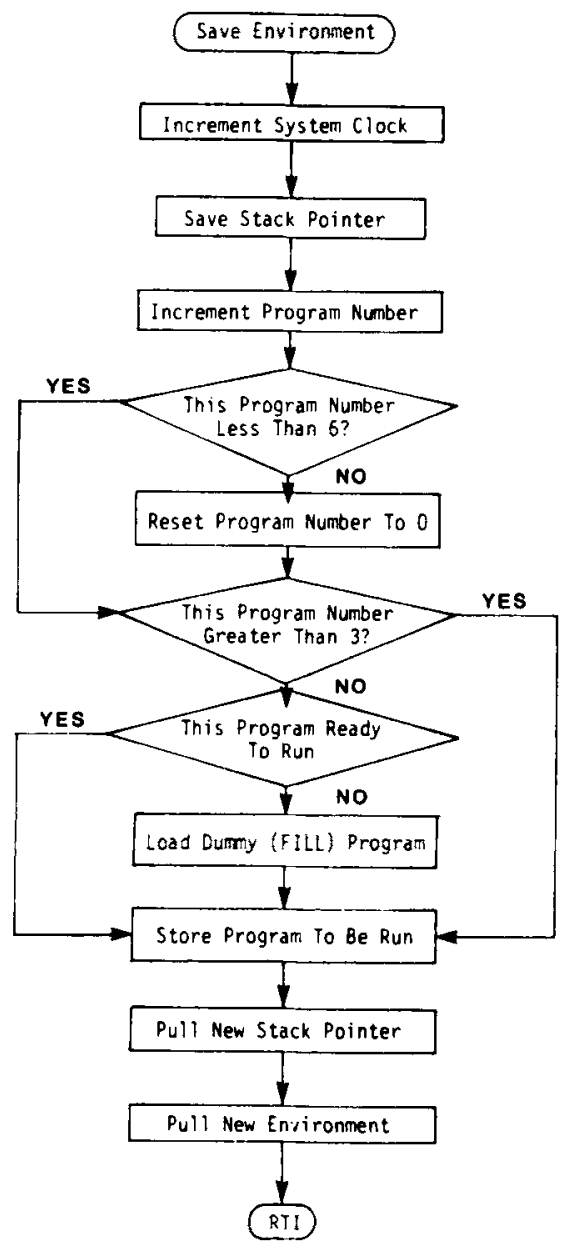

Figure 1. Flowchart of IRQ multitasking program. 
Table 6

Assembly Language Listing of IRQ Multiplexing Program

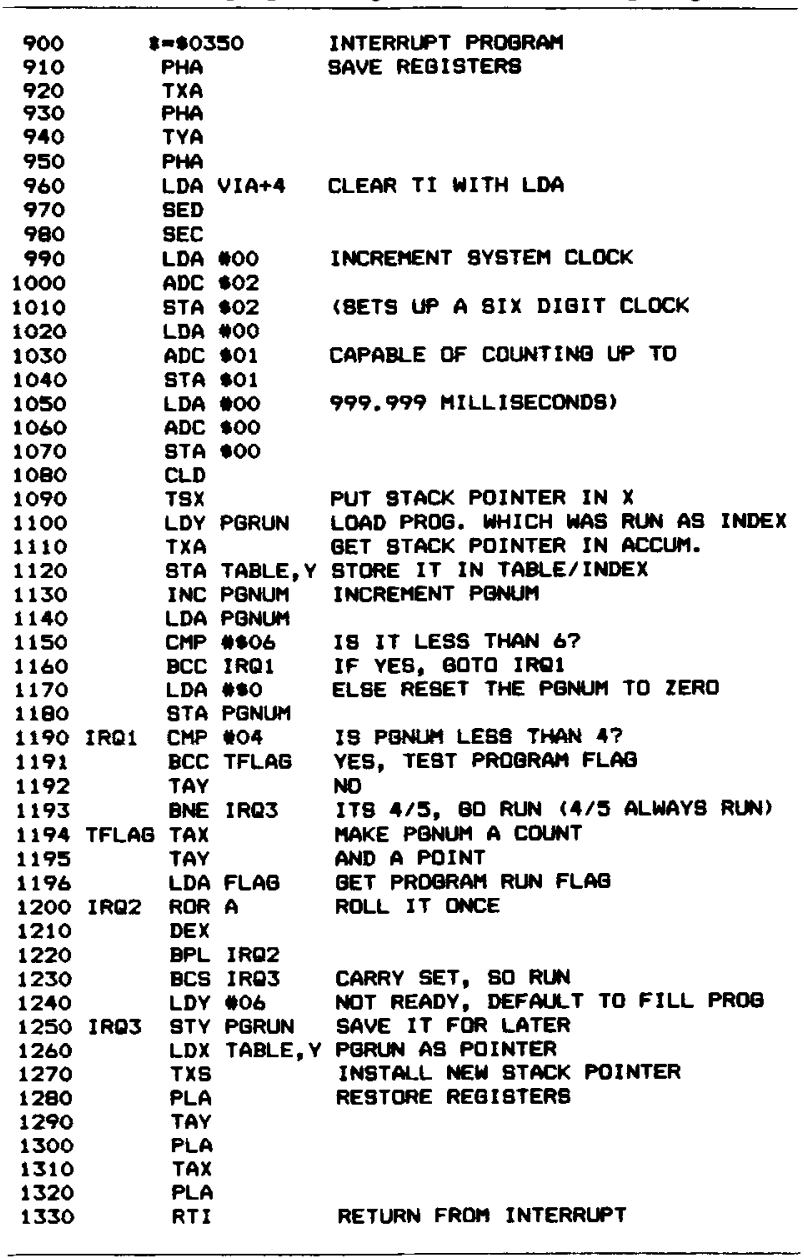

Table 7

Assembly Language Listing of Program for System Initialization

\begin{tabular}{|c|c|c|}
\hline $\begin{array}{l}10 \\
20 \\
30 \\
40 \\
50 \\
60 \\
70 \\
100 \\
90 \\
100 \\
110 \\
120 \\
130 \\
140 \\
150 \\
160 \\
170 \\
190 \\
190 \\
200 \\
210 \\
220 \\
230 \\
240 \\
250 \\
260 \\
270 \\
290 \\
290 \\
300 \\
310 \\
320 \\
330 \\
340\end{array}$ & 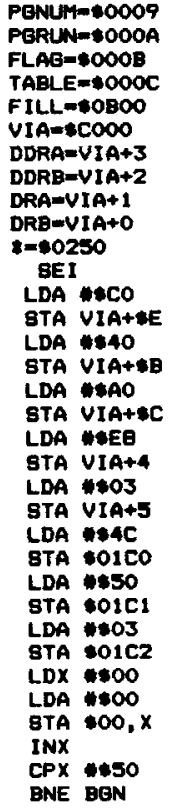 & $\begin{array}{l}\text { ENABLE T1 INTERRUPT } \\
\text { SELECT FREE-RUNNING MODE } \\
\text { ENABLE CB2 PLLSE MODE } \\
\text { LOAD COUNT INTO T1 } \\
\text { OO3EE=1000 (1/1000 EEC) } \\
\text { LOAD IRO VECTOR }\end{array}$ \\
\hline
\end{tabular}

more, each program must have its own unique area of memory for data storage, storage of flags, pointers, etc. Under certain circumstances, the separate programs (coroutines) may need to share information; consult Holtzer (1983) for a detailed discussion of this situation. The I/O capability of the microcomputer must also be divided among the separate programs, since each requires unique inputs and outputs (Arnold \& Hastings, 1984).

An important point in implementing multitasking is consideration of the bit slice duration, that is, how long each program or coroutine is allowed to run and what can be accomplished during that interval of time. In the example provided (Table 7), the $\mathrm{T} 1$ timer has been set up to generate an interrupt each millisecond. Thus, each coroutine can run for $1 \mathrm{msec}$ minus the time necessary to service the interrupt routine. The complete interrupt routine, as listed, requires slightly more than 100 micro$\mathrm{sec}$ for execution. Accordingly, the coroutine can run 900 microsec of each millisecond, or, at approximately 3 microsec per instruction, can execute around 300 instructions.

One final point should be emphasized at this time: In most studies involving behavioral parameters, the majority of the CPU's time will be spent in a loop waiting for an event to occur-waiting for a response to be made, for a reinforcement period to time out, etc. In actuality, probably no more than 20 and certainly fewer than 50 instructions-other than the looping operation-would ever be executed during any one slice duration. Furthermore, if the slice duration is $1 \mathrm{msec}$ and six programs are running, then the resolution of the system is $6 \mathrm{msec}$; that is, the maximum number of events that can be detected per coroutine per second is approximately 150 , which is sufficient for most all behavioral parameters.

In order to make this program completely functional, an initialization program is necessary to configure the VIA, clear memory, set up pointers, etc. (see Table 7).

\section{REFERENCES}

Arnold, N. D., \& Hastings, L. (1984). A bit-addressable I/O interface for microcomputers. Behavior Research Methods, Instruments, \& Computers, 16, 135-136.

Brown, W. (1981, October). A simple implementation of multitasking. Byte, 6, pp. 176-180.

Butler, F. E. (1980). MicroSKED. Behavior Research Methods \& Instrumentation, 12, 152-154.

Carroll, M. E., Santi, P. A., \& Rudelt, R. L. (1981). A microcomputer system for the control of behavioral experiments. Pharmacology, Biochemistry, \& Behavior, 14, 415-417.

Houtzer, J. (1983). Add multiple tasks to your communication and control program. Byte, 8, pp. 445-478.

Kaplan, H. L. (1980). Applications of multiprogramming software to real-time experiments in psychology. Behavior Research Methods \& Instrumentation, 12, 137-151.

Ternes, J. W., Ehrman, R., \& O'Brien, C. P. (1982). A software system for real-time control of psychological experiments. Behavior Research Methods \& Instrumentation, 14, 160-164.

Wyss, C. R. (1983, May). A conceptual approach to real-time programming. Byte, 8, pp. 452-470. 